\title{
Influence of maize genotype on rate of ruminal starch degradation
}

\author{
B Michalet-Doreau 1, M Champion 2 \\ IINRA, SRNH, Theix, 63122 St-Genès-Champanelle; \\ zLimagrain Genetics International, BP 1, 63720 Chappes, France
}

The digestibility of starch through the whole digestive tract is high in the ruminant, but the proportion of digestion achieved in the rumen widely varies (Harmon, 1992, J Nutr, 122, 203210 ). These varying degradation rates in the rumen can be attributed not only to the chemical nature of the starch itself, amylose or amylopectin, but also to the endosperm structure of the grain, and particularly the protein matrix in which starch granules are embedded (Kotarski et al, 1992, J Nutr, 122, 178-190). The objective of our study was to investigate how starch chemistry and structure within the grain matrix influence ruminal starch degradation.

The trial was performed on two maize cultivars characterised by variable proportions of vitreous and floury endosperm, with a protein matrix respectively high or low in cells. For each cultivar, we had three genotypes bearing the "waxy" gene to different degrees, a "waxy" starch, a "normal" starch and an "extender" starch. The amylose: amylopectin ratios were determined for the six samples and did not vary with the nature of endosperm. They were at mean 5:95, 46:54 and 75:25 for "waxy", "normal" and "extender"starchs, respectively. The 6 maize were ground to $2 \mathrm{~mm}$ mesh size, and their degradation rate in the rumen was measured by the nylon bag technique. The kinetics of starch degradation, adjusted to an exponential model, were analysed in a splitplot model, with texture endosperm as whole plot treatment and nature of starch as subplot.

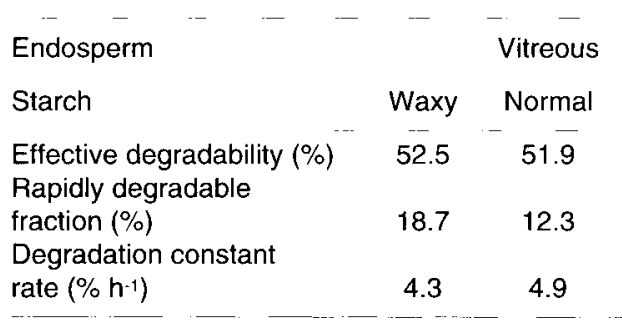

The degradability of the maize starch ranged widely, from 52.0 to $76.7 \%$. The starch in the floury cultivars was on average more degradable than that in the vitreous cultivars (71.0 vs 58.0 ), showing the protective role of the protein matrix surrounding the starch grains. Mc Allister et al (1993, J Anim Sci, 71, 205-212) showed in vitro the influence of nonstarch components on the different fermentation rates of starch between maize and barley. But the nature of starch also seems to be an important factor of variation. The "waxy" maizes were more degradable than the normal ones, at least when the endosperm is floury $(+10.8)$, and this increased degradability resulted essentially from a higher degradation rate. This effect cannot be ascribed only to their high amylopectin content. The "extender" maizes were the most readily degraded while their amylopectin content was lowest. This increased degradability was due to a greater rapidly degradable fraction. A starch-protein interaction would occur within cereal grains as observed by Streeter et al (1990, Anim Feed Sci Technol, 29, 279-287) with sorghum varieties differing in tannin level and starch nature, "waxy" or not. The increased degradability associated with the "waxy" genotype observed here could be due more to a modification of the structure of the endosperm, as shown by microscopy (Sullins and Rooney, 1975, Cereal Chem, $52,362-366)$, than to the biochemical nature of starch.

\begin{tabular}{ccccc} 
& \multicolumn{3}{c}{ Floury } \\
Extender & Waxy & Normal & Extender & SE \\
- & & - & $-\cdots$ & - \\
69.5 & 73.5 & 62.7 & 76.7 & 1.5 \\
27.3 & 21.0 & 26.7 & 50.7 & 5.1 \\
8.3 & 22.9 & 5.8 & 6.7 & 2.3 \\
\hline
\end{tabular}

